\title{
REPLY TO
}

\section{P. EBERT AND M. ROSSBERG'S FRIENDLY LETTER OF COMPLAINT}

EDWARD N. ZALTA

Center for the Study of Language and Information

Stanford University

Stanford, CA 94305

USA

Subject: Reply to P. Ebert and M. Rossberg's friendly letter of complaint

Dear Philip and Marcus,

Thanks very much for your interesting letter. I, too, enjoyed the time we spent doing philosophy at the Kirchberg meetings. I think we furthered our understanding of each other's views, especially during the workshop on neologicism and during the extended dinner conversations in the evenings.

Let me reply to your criticisms of my neologicist views, many of which I've developed with co-author Bernard Linsky. I've consulted with Bernie about your letter. From my reading of it, I found 9 critical conclusions. But let me preface my replies to specific criticisms with some context.

When I first developed the theory of abstract objects and modeled mathematical objects in the theory, I assumed abstract objects exist independently of us in some special domain and that we get knowledge of them via some kind (or faculty) of intuition. I assumed that the best we could do as philosophers would be to use mathematical rigor to systematize this domain by way of a group of axioms that included a single existence principle that comprehended the domain. But I've subsequently come to believe that epistemological considerations require a more refined view of the mind-independent existence and objectivity of abstract objects, and of the axiom that comprehends them. Linsky and I (1995) argued that one cannot apply the usual model of the mind-independence and objectivity of con- 
crete objects to explain knowledge of abstract objects. ${ }^{1}$ A different model is called for, and we described it at some length in our paper.

I suspect you have a different understanding of the mind-independence and objectivity of abstract objects, and it is not clear to me that it matches up with the objectives of the neologicist project, whether yours or ours. You don't provide, for example, a conception of abstract objects in general, but rather insist that they are the kind of thing that we can come to know on the basis of certain Fregean biconditionals and the existence of the equivalence relations on which they are based. I think this is in tension with your view that neologicism implies a single theory of extensions. Linsky and I argued that abstract objects are defined by our conceptions of them and that a plenitude principle, like the comprehension principle I've developed (which yields a different abstract object for each different conception) is what is needed to put logicism and Platonism on a sound (epistemological) basis. And a plenitude principle that encodes the properties connected with each distinct conception into a distinct abstract object yields not only an understanding of the nature of abstract objects, but explains why mathematicians and story-tellers have wide latitude and a great deal of conceptual freedom when they bring their imaginative powers into play as they develop mathematical theories and tell stories (modulo certain crucial differences of course, to be described below). From this point of view, one will be less likely to suppose that there is a single, true theory of sets, for example.

I therefore think it is a mistake to suppose that mathematicians are in the business of determining, for each pair of competing theories, whether one is true and the other false. Instead, one should think of mathematicians as interested in (a) systematizing conceptions of mathematical objects and relations in the most powerful and interesting way, and (b) finding proofs of claims that follow from those conceptions once the governing principles are clear. When Gauss, Lobachevsky, Bolyai, and Riemann became interested in geometries in which the sum of the three angles of a triangle is less (or more) than $180^{\circ}$ (or more generally, in which Euclid's fifth postulate is replaced with one of its denials), the truth or falsity of those alternative theories wasn't at stake; rather what was at stake was the interest, power, and applicability of the theorems implied by the different conceptions of point, line, triangle, etc. that could be used to develop spherical and hyperbolic geometries. Similarly, though it may be that Weierstrass thought that

${ }^{1}$ See 'Platonized Naturalism versus Naturalized Platonism', Journal of Philosophy, XCII/10 (October 1995): pp.525-555. 
analysis formulated with infinitesimals was 'false', what he did was to systemize a conception of analysis that eliminated infinitesimals and on which the relevant concepts and theorems of analysis become defined and derived from the definition of a limit. That didn't falsify analysis based on infinitesimals, given Robinson's work, but rather founded analysis on a different, more rigorous, conception than the one available at the time.

As to set theory, I don't think that the issue before Cantor, Zermelo, Fraenkel and the other early set theorists was to refute one or another set theory; the problem was to show that one could systematize conceptions of 'set' and 'membership' in ways that avoided the paradoxes, and show that the resulting theory was a more powerful systematization of those notions. To put it bluntly, I think it would be philosophically naive for a set theorist like Woodin to claim that Aczel's nonwellfounded set theory is just 'false'. Such a claim is not philosophically justified, and indeed, just misses the point. If set theorists do use the language of truth and falsity (if not in connection with Aczel, then in connection with extensions of ZFC employing definable determinacy or large cardinals) to describe competing theories, philosophers would be better served to characterize the debate as a disagreement about the way to develop the most powerful conception of 'set' and 'membership'. Each set theory embodying a different conception of these notions should be seen as characterizing a distinctive domain. And so on for other mathematical theories, applied or unapplied.

With this in mind, I can reply briefly to your critical conclusions.

1. The analysis of mathematical statements Zalta defends turns the categorical statements of mathematics into non-categorical 'hedged' statements.

2. Even if he were to drop the 'theory operator' from the analysis of each mathematical claim, the resulting statement still wouldn't be categorical because the terms are indexed to their respective theories.

I am not sure I understand the definition of 'categorical' you are using. To take an example, consider the language of $\mathrm{ZF}$ and the claim that "the null set has no members", expressed in that language. I've analyzed this claim, in the first instance, as the following truth about ZF:

$$
\mathrm{ZF}=\left[\lambda y \neg \exists z\left(z \in \in_{\mathrm{ZF}} y\right)\right] \varnothing_{\mathrm{ZF}}
$$


which asserts that, in the theory ZF, the null set of ZF exemplifies the property of having no members (where the membership relation is the one characterized by the theorems of ZF). ${ }^{2}$ This is a categorical claim about the theory ZF. Moreover, given the ambiguity I postulate in natural language predication, I also provide two readings of the unadorned claim "the null set has no members", one true and one false, as follows:

$$
\begin{array}{ll}
\varnothing_{\mathrm{ZF}}\left[\lambda y \neg \exists z\left(z \in \in_{\mathrm{ZF}} y\right)\right] & \text { (true) } \\
{\left[\lambda y \neg \exists z\left(z \in \in_{\mathrm{ZF}} y\right)\right] \varnothing_{\mathrm{ZF}}} & \text { (false) }
\end{array}
$$

The first asserts that the null set of ZF encodes the property of having no members, while the latter asserts that the null set exemplifies the property of having no members. Both readings are categorical (they are both atomic formulas with a complex predicate), and the former one preserves the intuition that mathematicians have asserted something true with this claim. The fact that they talk about the particular object "the null set of ZF" and the particular relation "the membership relation of ZF" doesn't undermine the categoricity of the claims. Are these claims 'hedged' in some illegitimate way? Well, only if you suppose that there is a single, true theory of sets and the membership relation. That is something I deny, for the reasons mentioned above.

3. The view Zalta defends doesn't entail an epistemically relevant difference between mathematical knowledge of a consistent theory and 'knowledge' of an inconsistent theory.

To answer this criticism, consider an example. Although we can detect the inconsistency in Frege's system syntactically, nevertheless, when you pick up a copy of Frege's Grundgesetze you come to understand its language because the terms of his language have a semantic significance and the sentences in the Grundgesetze are meaningful. I further believe that the way we come to know the significance of the terms, and meaningfulness of the sentences, deployed in Frege's theory is just like the way we come to know the significance of the terms, and meaningfulness of the sentences, deployed in other mathematical theories. So, in fact, in some deep sense, there is no epistemically relevant difference in the way we come to understand, and have knowledge of, consistent and inconsistent theories.

${ }^{2}$ The statement ' $\mathrm{ZF} F p$ ' is defined in object theory to be: $\mathrm{ZF}[\lambda y p]$, i.e., $\mathrm{ZF}$ encodes the property being such that $p$. 
But object theory does imply there is a difference between the two kinds of theories. In the former, the terms denote objects that are typically incomplete with respect to their encoded properties, i.e., objects $x$ which are such that, relative to the properties $F$ expressible in the language of the theory, $x$ neither encodes $F$ nor encodes the negation of $F$. But the terms of inconsistent theories denote objects that encode every property expressible in the language of the theory. The objects denoted are uninteresting by comparison. For example, the empty extension of Frege's Grundgesetze encodes every property $F$ expressible in the language of Grundgesetze, since it is defined as the object that encodes exactly the properties $F$ such that, in the theory of the Grundgesetze, the empty extension exemplifies $F$. Given the inconsistency in Grundgesetze, the empty extension of that theory encodes every property. Note that this does not trivialize the meaning of the expressions in Frege's language as long as you distinguish the sense and reference of those expressions. Though the denotations of the terms (predicates) in Frege's system become analyzed, on the above scheme, as abstract objects (relations) that encode every property expressible in the system, the senses of these expressions should not be so analyzed. The senses are abstracta that encode just the properties that strike our cognitive understanding as we come to understand Frege's system, and given that Frege himself didn't recognize that there was an inconsistency when he proposed his axioms, we might suppose that the senses of the terms are consistent (i.e., encode properties that are jointly consistent). So, when we discover that a theory is inconsistent, we learn that the objects denoted by the terms of the theory have (in the encoding sense) every property expressible in the language of the theory.

4. The view Zalta defends doesn't entail that the difference between mathematical knowledge and knowledge of fictions is substantive.

I'm not sure what 'substantive' means in this context. Clearly, we do have knowledge of fictional entities. We know the ancient Greeks worshiped Zeus, that Holmes is more famous than Pinkerton, etc. Does the fact that a theory treats fictions and mathematical objects both as abstract objects (and identified relative to their background conceptions) mean that there is no substantive difference in our knowledge of these entities? I don't think so. For one thing, stories are objects that are closed under relevant entailment, not logical entailment, whereas mathematical theories are closed under 
logical entailment, not relevant entailment. That seems like a substantive difference in the way we conceive of these objects, and thus a substantive difference in the way we come to know them. But more importantly, theoretical mathematical objects encode only abstract relations (relations which are themselves subject to the analysis of higher-order object theory), whereas fictional objects typically encode ordinary relations. ${ }^{3}$ The exceptions to this are stories involving fictional relations (e.g., the property of being a hobbit in The Lord of the Rings). Fictional relations are analyzed in object theory as abstract relations that encode properties of relations. Note that the properties of relations encoded by being a hobbit, such as being a species in which the individuals are human in form, with legs, arms, hairy feet, etc., involve ordinary properties. By contrast, the object-theoretic identifications of abstract mathematical relations, like less than, membership, group addition, etc., involve properties of relations that never reference ordinary properties. This strikes me as a substantive difference between fictional relations and mathematical relations.

5. Logicism implies that there is a single theory of sets and thus, by extensionality, a unique empty set, whereas the analysis Zalta offers implies that there is a different empty set for each distinct mathematical theory of sets.

Although I replied to this criticism in the preface, let me say that I'm not sure that it is true that logicism implies that there is a single empty set. Russell and Whitehead were logicists, but their theory implies there is an empty set at each type. And should we also believe that logicism implies that there is a single theory of numbers? That seems clearly false: Frege held that the natural numbers were different from the real numbers; he used different symbols for them: $Q, 1, \ldots$ for the naturals and $0,1, \ldots$ for the reals. If logicism were to imply all these things, I think it would be false, since there is no single, true theory of sets and no single, true theory of numbers. There are as many different theories of sets and numbers as there are con-

\footnotetext{
${ }^{3}$ I distinguish here 'theoretical' mathematical objects, like the null set of ZF, from 'natural' mathematical objects, like the number of planets, the class of humans, the direction of line $a$, etc. See my 'Essence and Modality', Mind, 115/459 (July 2006): pp.659-693. Natural mathematical objects are not identified relative to a background story or theory, but can be defined in object theory using logical notions and distinguished equivalence relations. These natural mathematical objects may indeed encode ordinary properties: the number of planets, for example, encodes the property of being a planet.
} 
ceptions of 'set' and 'number'. Fortunately, I don't think logicism does imply there are single, true theories of sets and numbers.

6. The view Zalta defends entails that there are no genuine disputes about the truth of a mathematical theory.

7. Since my view of what counts as acceptable mathematical theories is liberal and would not appeal to Frege's logicist way of thinking, it can't be counted as neologicist (or logicist).

I've already responded to (6) in the preface to my replies. As to (7), I think the practice of mathematics establishes that we must be liberal as to what counts as a mathematical theory. The range of mathematical theories and the freedom mathematicians have to innovate is clear by inspection. The suggestion that one can reduce, by way of relative interpretability, all mathematical theories to logic strikes me as false, and if the early logicists believed in such a reduction, then they had the wrong notion of reducibility. If relative interpretability is the standard of reduction, then logic must already include the power of mathematics if arbitrary mathematical theories can be reduced to it. But there is no conception of logic I know of on which it does have this power.

8. The view Zalta defends is not neologicist (or logicist) because it doesn't achieve Frege's aim to prove theorems within logic to reduce the enterprise of mathematics to logic.

I think we are now returning to the core of our differences. For the reasons just mentioned, I believe Frege and the early logicists were using the wrong notion of reduction. I see no hope for a successful logicist or even neologicist reduction of mathematics to logic if relative interpretability is the standard of reduction. (Kit Fine has given us a good idea of what the limits of abstraction are for neologicism.) While it is true that Frege aimed to prove theorems within logic to reduce mathematics to logic, it is important to recognize that his aim was motivated by a larger strategy, namely, that of explaining how we grasp or apprehend mathematical objects without appealing to (some faculty of) intuition. I think we can achieve the goal of Frege's larger strategy by reducing mathematics to logic using a different standard of reduction, i.e., in terms of ontological reducibility instead 
of relative interpretability. ${ }^{4}$ Third-order object theory gives us the means to analyze both the individual terms and predicates of arbitrary mathematical theories in terms of canonical descriptions of abstract individuals and abstract relations. And object theory gives us the means to produce categorical, and indeed provably true, readings of the theorems of mathematical theories. These readings, and the descriptions used to express them, all derive from a single abstraction principle, which has a legitimate claim to being called 'analytic'. That addresses Frege's larger goal of eliminating appeals to (a faculty of) intuition when apprehending mathematical objects, and it does so in a way that Frege might endorse, namely, in terms of a single analytic principle added to logic.

9. The view Zalta defends is not neologicist (or logicist) because he hasn't offered an epistemology that shows how mathematical knowledge is based on logical knowledge.

Bernard Linsky and I offered an answer in our 2006 paper $^{5}$ and I developed it further in my talk at the Wittgenstein Symposium. Let me illustrate the answer with an example. Consider the ZF statement "the null set has no members," which (we saw above) has a true reading: $\varnothing_{\mathrm{ZF}}\left[\lambda y \neg \exists z\left(z \in \in_{\mathrm{ZF}} y\right)\right]$. This true reading turns out to be a theorem of object theory and its derivation rests on:

- Third-order logic with a relational $\lambda$-calculus

- (Second-order) Abstraction for abstract individuals $x(A ! x \& \forall F(x F \equiv \phi)) G \equiv \phi G, F$

- (Third-order) Abstraction for abstract relations $\imath R(A ! R \& \forall F(R F \equiv \phi)) S \equiv \phi S, R$

- The analytic truth "According to ZF, the null set has no members," which is imported into object theory receiving the analysis described above.

I think the axioms of third-order logic are analytically true, since logically true. Using second-order logic under extremely small, general models (in which $\lambda$-conversion, i.e., the $\beta$-reduction principle of the $\lambda$-calculus, is log-

\footnotetext{
${ }^{4}$ The notion of ontological reduction was illustrated in my paper 'Neologicism? An Ontological Reduction of Mathematics to Metaphysics', Erkenntnis, 53/1-2 (2000): pp.219-265.
}

${ }^{5}$ See 'What is Neologicism?', Bulletin of Symbolic Logic, 12/1 (2006): pp.60-99. 
ically true) as an analogy, I argued that the abstraction principles for abstract individuals and abstract relations (which are themselves true in extremely small models) are similarly analytically true, since logically true. Finally, I argued that the claim "According to ZF, the null set has no members" is analytically true. We need no special faculty of intuition to know these four classes of analytic truths. And since the theorems derived from analytic truths are also analytic, I conclude that the claim "the null set has no members", under its true encoding reading, is also analytic and thereby knowable. ${ }^{6}$

This ends my replies. I've enjoyed our exchange and look forward to our future discussions about these issues.

Respectfully yours,

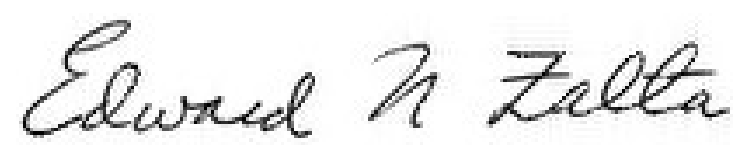

Edward N. Zalta

Senior Research Scholar zalta@stanford.edu

\footnotetext{
${ }^{6}$ This complements what Linsky and I argued in 1995 (op. cit.), namely, that knowledge of mathematical objects and mathematical relations is by description, since each mathematical object and relation is identified in object theory using a canonical description. Indeed, we argued that knowledge by acquaintance and knowledge by description collapses in the case of abstract objects.
} 
